ARTICLE

\title{
Geant4 Simulation to Study the Sensitivity of a MICRON Silicon Strip Detector Irradiated by a SIEMENS PRIMUS Linac
}

\author{
Miguel A. CORTÉS-GIRALDO ${ }^{1, *}$, María Isabel GALLARDO ${ }^{1}$, Rafael ARRÁNS ${ }^{2}$, José M. QUESADA ${ }^{1}$, \\ Alessio BOCCI ${ }^{3}$, José M. ESPINO ${ }^{1}$, Ziad ABOU-HAÏDAR ${ }^{3}$ and Marcos A. G. ÁLVAREZ ${ }^{1}$ \\ ${ }^{1}$ Departamento de Física Atómica, Molecular y Nuclear, University of Sevilla, 41080 Sevilla, Spain \\ ${ }^{2}$ Servicio de Radiofísica, H. U. Virgen Macarena, 41007 Sevilla, Spain \\ ${ }^{3}$ Centro Nacional de Aceleradores (CNA), 41092 Sevilla, Spain
}

\begin{abstract}
A Geant4 application has been developed to simulate the energy deposited in a silicon strip detector, model W1(SS)500, manufactured by Micron Semiconductor Ltd., irradiated with a Siemens PRIMUS ${ }^{\mathrm{TM}}$ linac dual energy machine, operating at 6-MV photon mode. The goal of these simulations is to estimate the sensitivity of this detector in different situations, according to the energy deposited in each strip. The application was compiled using Geant 4 Monte Carlo code (version 9.3.p01). It includes the modelled geometry of the Siemens PRIMUS linac treatment head, incorporating shielding and Multi-Leaf Collimator (MLC), a detailed model of a W1(SS)-500 Micron silicon strip detector and two different phantoms: one made of $30 \times 30 \mathrm{~cm}^{2}$ water-equivalent slabs, and another having a quasi-anthropomorphic phantom (15-cm diameter cylinder) made of polyethylene. In order to calibrate the simulation results, we compare Geant 4 calculations of delivered dose in a water tank with experimental measurements in reference conditions (sourceto-surface distance $S S D=100 \mathrm{~cm}, 10 \times 10 \mathrm{~cm}^{2}$ field). With the detector placed within the water-equivalent phantom, perpendicularly to the beam direction, we calculate the average dose in each strip of the detector in two situations. First, reference conditions with detector placed at depth $z=1.5 \mathrm{~cm}$. Second, half-field conditions (one side of the MLC aperture set to zero) with phantom at $S S D=90 \mathrm{~cm}$ and $z=10 \mathrm{~cm}$ was simulated. The Monte Carlo simulations considering the cylindrical phantom recreate conditions closer to those normally found in clinical environments. In this case, the phantom is placed at isocenter with the detector plane oriented parallel to beam direction. Different rotation angles of the phantom were considered in order to study the detector performance in different orientations. These calculations were compared with results obtained by a Philips Pinnacle treatment planning system (TPS).
\end{abstract}

KEYWORDS: GEANT4, radiotherapy, IMRT, verification

\section{Introduction}

The increasing sophistication and complexity of IntensityModulated Radiation Therapy (IMRT) treatments is a major challenge for treatment planning systems (TPS) which might miscalculate under some circumstances. ${ }^{1,2)}$ This is the reason why empirical dose distribution verification is highly advisable prior real dose delivery to patient. The spatial resolution of the so called 2D arrays (either based on ion chambers or diodes) is still far from that needed in treatment verification. On the other hand, film dosimetry is extensively accepted as $2 \mathrm{D}$ dosimeter ${ }^{3)}$ and there are some extensive reviews on the use of radiochromic films as a dosimeter. ${ }^{4,5)}$ However, its intrinsic measuring and reading process makes it unsuitable as on line detector. An excellent alternative might be the silicon microstrip technology, but a high number of allocated channels and complex multichannel readout electronics are then needed to obtain the necessary submillimetre spatial resolution. ${ }^{6)}$

At therapy energies the cross section is dominated by

*Corresponding author, E-mail: miancortes@us.es

(C) 2011 Atomic Energy Society of Japan, All Rights Reserved.
Compton interactions, and consequently we are dealing with non-local contributions of secondary electrons; therefore, it is necessary to place the detector under electronic equilibrium conditions within the phantom used for dosimetry studies. ${ }^{7}$ ) According to this, we have designed two phantoms to house a single sided silicon strip detector, so that electronic equilibrium conditions were satisfied and the perturbation caused by the detector in the behaviour of the phantom under irradiation was minimized. To study the dosimetric behaviour of the detector, one of the phantoms is made of water-equivalent slabs. In turn, to verify the $2 \mathrm{D}$ treatment dose maps, another phantom, made of polyethylene, presents quasi-anthropomorphic shape and has the capability of rotating along its symmetry axis. Both materials have densities similar to those for human tissues and are suitable for dosimetry studies.

This work is included in a more ambitious project aiming to increase the geometrical precision of a silicon strip detector with a discrete readout electronics by means of an in-house algorithm (patent pending). In particular, this contribution presents the simulation of the performance in the phantom of a commercial totally depleted DC-coupled single-sided silicon 
strip detector, manufactured by Micron Semiconductor Ltd., covering an active area of $50.0 \times 50.0 \mathrm{~mm}^{2}$ and divided into 16 narrow strips with $3.1 \mathrm{~mm}$ pitch and $500 \mu \mathrm{m}$ thick each. The usefulness of Monte Carlo (MC) simulation is clear in order to figure out the influence of the different factors involved in the measuring process.

Thanks to the increasing power in computing capabilities and software development, the use of MC techniques has become a standard tool in recent years for the computer simulation of the clinical components of linear accelerators, notably beam generation and collimation, and for the interaction of radiation beams with patients and dosimetric materials. MC methods are also increasingly used for radiotherapy treatment planning and dose quality assurance of the dose delivery at clinical and research centres worldwide. Extremely accurate simulations of detector response to accelerator-produced beams are being conducted today for dosimetric purposes. Geant $4^{8,9)}$ - a full object-oriented toolkit for radiation transport through matter - is a general purpose Monte Carlo code that offers an advanced framework for such simulations. Geant 4 toolkit was created for applications in high-energy physics, but nowadays it is used in many other fields, e. g. nuclear physics and space applications. Geant4 validation for medical applications has been undertaken by different groups, ${ }^{10-14)}$ and it has also been widely used in proton therapy applications. ${ }^{15,16)}$

This work constitutes an approach to silicon strip technology to study the pros and cons of using this kind of detectors in clinical conditions and provides a method to find the most appropriate characteristics (frame material, strip thickness, etc.) to be used as 2D treatment verification system.

\section{Method}

Monte Carlo simulation have been performed in two steps. First, Siemens PRIMUS ${ }^{\mathrm{TM}}$ clinical accelerator, with nominal photon energy of $6 \mathrm{MV}$, has been modelled to fully characterize its dosimetric behaviour. This step consists of the modelling of all the elements within the treatment head including a detailed simulation of the double-focused Multi-Leaf Collimator (MLC).

Then, once the simulation of the accelerator leads to a good matching with the empirical dosimetry measurements, such as Percentage Depth Dose (PDD) curves and dose profiles perpendicular to the beam axis for various depths, the new simulation process deals with the geometry of a single-sided silicon strip detector, covering an active area of $50.0 \times 50.0 \mathrm{~mm}^{2}$ and divided into 16 narrow strips with $3.1 \mathrm{~mm}$ pitch and $500 \mu \mathrm{m}$ thick each. This part of the work is two-folded:

On one hand, several relevant dosimetry curves have been simulated in a water-equivalent slab phantom with a special housing to accommodate the detector perpendicular to the beam axis (Fig. 1). These simulations were:

- Profile calculated considering the detector placed at $100+1.5 \mathrm{~cm}$ (phantom source to surface distance, $S S D$, equal to $100 \mathrm{~cm}$; detector depth, $z$ equal to $1.5 \mathrm{~cm}$ ), irradiated by a $10 \times 10 \mathrm{~cm}^{2}$ standard field. With calibration purposes, these calculations are compared to those obtained by considering a water tank at $S S D=100 \mathrm{~cm}$.

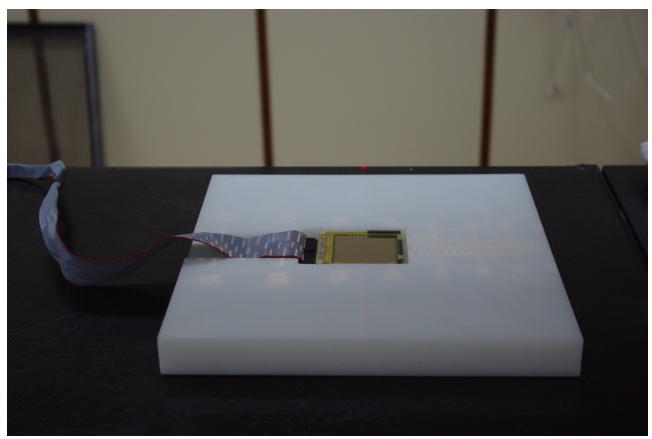

Fig. 1 A detail of the slab phantom housing the detector.

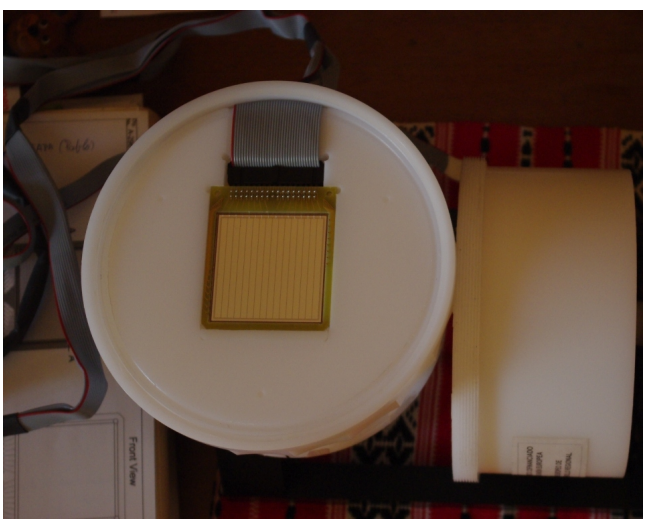

Fig. 2 Cylindrical phantom showing the detector at the axial plane.

- A profile of half beam $\left(5 \times 10 \mathrm{~cm}^{2}\right)$ at $90+10 \mathrm{~cm}$ $(S S D=90 \mathrm{~cm}, z=10 \mathrm{~cm})$ to estimate the penumbra. The width of the penumbra obtained with silicon strip detector is compared with the same value calculated in a water tank.

On the other hand, taking into consideration that the most common way to present dose distributions in radiotherapy is a dose map in the axial plane of the patient (i.e. parallel to the beam axis), several simulations have been made with a $10 \times 10$ $\mathrm{cm}^{2}$ beam with the detector plane parallel to the beam axis and varying the angle of the strips with respect to it. The detector is placed in a quasi-anthropomorphic cylindrical phantom made of polyethylene which can rotate along its symmetry axis (Fig. 2). These simulations aim to check the behaviour of the detector at any orientation in the patient's axial plane.

Monte Carlo results for the water-equivalent slab phantom are compared with data measured by an ion chamber to get a first calibration factor for the detector, as explained above. The calculations obtained with the cylindrical phantom were analysed with respect to those of the Philips Pinnacle treatment planning system (TPS) in order to verify whether an extra angle-dependent calibration factor is needed. Experimental data measured with the detector are in progress.

\section{Geant4 Simulation}

Geant4 provides several physics settings through its physics lists. ${ }^{17)}$ In our simulations, the so called Livermore Low-Energy electromagnetic physics lists is consid- 


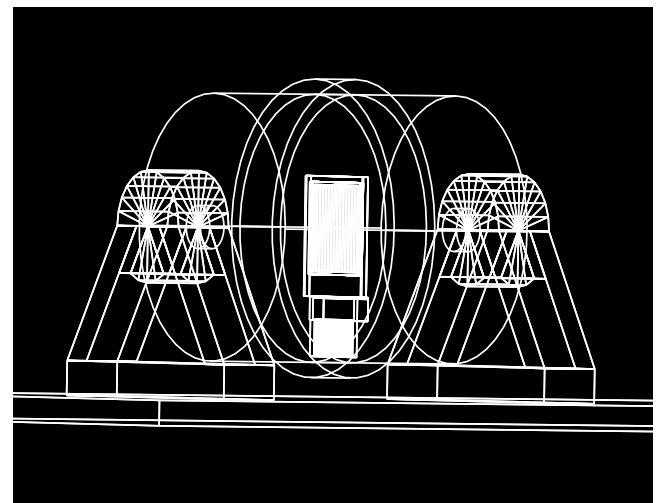

Fig. 3 The quasi-anthropomorphic cylindrical phantom modelled with Geant4 visualized with HepRepApp tool.

ered. Geant4 Livermore package ${ }^{18,19)}$ extends its application range down to $250 \mathrm{eV}$. Rayleigh scattering and atomic relaxation processes are implemented in this package. These models implement cross-section tables obtained from the Lawrence Livermore National Library, including evaluated data for photons, ${ }^{20)}$ electrons ${ }^{21)}$ and atoms. ${ }^{22)}$ Since no data for positrons are present, Geant4 Standard EM physics list ${ }^{23}$ ) is used in this case. The production cuts were set to $0.1 \mathrm{~mm}$ for all kind of particles in all regions. This means that every secondary particle created will not be explicitely considered when its expected range in the material is below this value. In these cases, Geant 4 considers the energy lost by the primary particle as delivered locally at the point of interaction. This setting is significantly smaller than the sensitive volume of the detector model, thus ensuring the accuracy of the spatial distribution of the energy deposited in the simulation.

All the Geant4 MC calculations presented in this work have been performed in a linux cluster (Ubuntu Server 8.04 64bit and Debian Etch 64-bit) with three Dell ${ }^{\mathrm{TM}}$ PowerEdge ${ }^{\mathrm{TM}}$ 2970 servers, with two quad-core processors Intel ${ }^{\circledR}$ Xeon ${ }^{\circledR}$ at $2.66 \mathrm{GHz}$ and $8 \mathrm{~GB}$ RAM memory each.

Our Geant4 application (version 9.3.p01) includes a detailed model of the Siemens PRIMUS linac treatment head with a shielding for electrons, photons and positrons. ${ }^{24)}$ Further, the geometry of both water-equivalent slab phantom and quasi-anthropomorphic cylindrical phantom was modelled using Geant 4 capabilities for solids. Figure 3 shows a scheme of the cylindrical phantom where the silicon strip detector is placed at the centre. The horizontal disposition of the cylinder, along the patient couch, and the lateral supports, in both sides, can be distinguished.

The Geant4 simulations were performed using IAEA phase-space (phsp) files created by means of the code developed by our group. ${ }^{25)}$ A phsp file stores the energy, position and momentum direction cosines of each particle crossing a plane perpendicular to the beam direction at a given distance $z$ from the source.

The MC simulations were realized in three steps:

1. The first step was devoted to characterize the fluence of particles going out of the motionless part of the treatment head (target, collimator, flattening filter and mon-

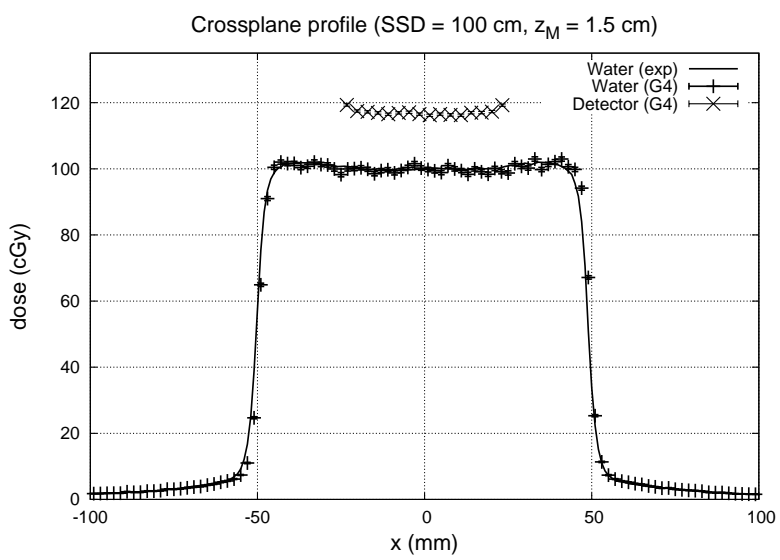

Fig. 4 Dose profiles (crossplane) for a $10 \times 10 \mathrm{~cm}^{2}$ field, $S S D=100 \mathrm{~cm}$ and $1.5 \mathrm{~cm}$ depth in a water tank (100 MU). Both Geant 4 calculations (water tank and phantom with detector) are normalized to experimental data (solid line) by assigning $100 \mathrm{cGy}$ to the dose at the profile centre, $d_{M}$, in the water tank simulation. The error bars represent the statistical uncertainties at $1 \sigma$ level. The effect of higher density and higher mean $Z$ of the detector material (silicon) can be clearly appreciated in the value of the dose.

itor chamber). With this purpose, a phsp plane was defined below the monitor chamber, and an IAEA phsp file was created by simulating $2 \times 10^{9}$ primary electrons (or histories) inciding on the target.

2. The IAEA phsp file created was used as the generator of primaries in this part of the simulation. Here, the transport of particles through the jaws and MLC was simulated and a second IAEA phsp file storing the information of the radiation field at $S S D=70 \mathrm{~cm}$ was generated. Each particle of the first phsp file was recycled 10 times to increase statistics. For recycling, it was taken into account that the first phsp file presents rotational symmetry with respect to the beam propagation axis, since the motionless part of the treatment head presents this symmetry. Thus, each particle was rotated randomly around the propagation axis prior creating the primary vertex, so that the variance in the second IAEA phsp file could be reduced.

3. Finally, the second IAEA phsp filed was used as the primary generator in the Geant 4 simulation of the irradiation of the water tank and the phantoms. Each particle was repeated 5 times for water tank simulations, and 20 times for both phantoms.

\section{Results}

In order to verify the Geant4 simulations, we have performed calculations of the dose profile at reference conditions: a $10 \times 10 \mathrm{~cm}^{2}$ field irradiating a water tank $(50 \times 50 \times 40$ $\mathrm{cm}^{3}$ ) at $S S D=100 \mathrm{~cm}$, dose profile measured at $z=1.5$ $\mathrm{cm}$. The Geant 4 calculations are compared with experimental data obtained with the ionization chamber. Figure 4 represents crossplane profiles obtained in these reference condi- 


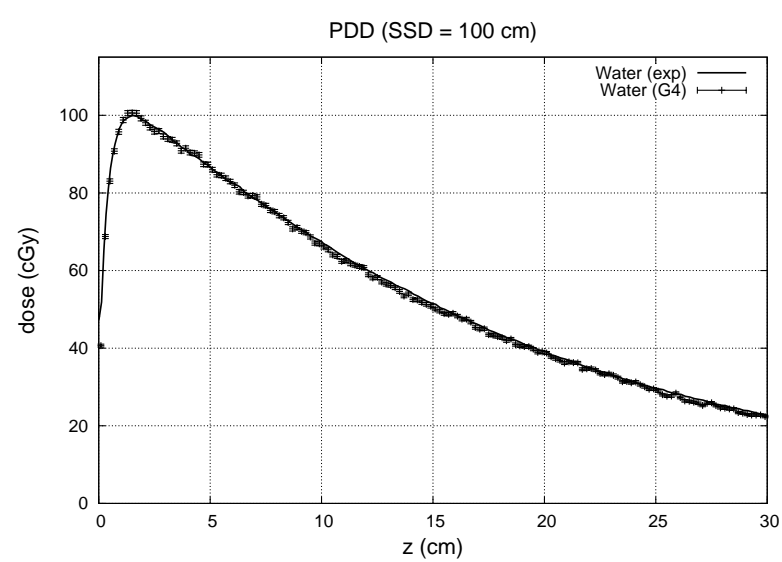

Fig. 5 Experimental (solid line) and Geant4-calculated (points) depth-dose curves. Geant 4 calculations were normalized with the same factor as in Fig. 4 in order to compare with absolute dose. The error bars correspond to the statistical uncertainty at $1 \sigma$ level.

tions. The experimental data represent the dose corresponding to 100 Monitor Units (MU), where MU is a common and arbitrary unit of fluence in a clinical accelerator which corresponds to a dose equal to $1 \mathrm{cGy}$ in the centre of a crossplane profile in these reference conditions. Geant 4 calculations represent the average dose per primary electron inciding the target; in other words, with Geant4 calculations we obtain the average dose per original history. The statistical uncertainty, obtained with the method discussed by Walters et al., ${ }^{26)}$ is approximately $0.9 \%$ ( $1 \sigma$ level $)$ in the central region. The central value of the dose profile $\left(d_{M}=1.085 \times 10^{-16} \mathrm{~Gy} / \mathrm{hist}\right)$ is then normalized to $100 \mathrm{cGy}$ in order to match the experimental results. With this normalization we achieve an agreement within experimental uncertainties in position $( \pm 1 \mathrm{~mm})$, whereas the dose calculated with Geant 4 agreed within $\pm 3 \%$ of the value of the experimental dose.

The silicon strip detector at reference conditions within the water-equivalent slab phantom was simulated with Geant 4 as well. The results were normalized with the factor obtained in the previous simulation. In Fig. 4 we can see that the average dose in the detector strips is approximately $20 \%$ higher than both measurements in the water tank with the ionization chamber detector and the normalized Geant 4 calculations in the water tank. This effect is due to the material composing the detector sensitive volume (silicon), which has higher density and higher $Z$ than water. For this silicon strip detector, we obtained $d_{M, S i}=1.266 \times 10^{-16} \mathrm{~Gy} / \mathrm{hist}$ for the central value of the profile. The value of the statistical uncertainty is $0.3 \%$, approximately. This result was used to re-calibrate the dose in the silicon detector to equivalent dose in water.

Figure 5 plots both depth-dose curves measured with the ionization chamber detector and calculated with Geant4. The symbols are the same as in Fig. 4. Statistical level of the Geant 4 simulation is the same as in the previous case $(1 \%$, $1 \sigma$, around the region of maximum dose), and it can be observed that the Geant 4 calculations agree with the experimental measurements within statistical uncertainties.

To study the response in the penumbra region, a half-

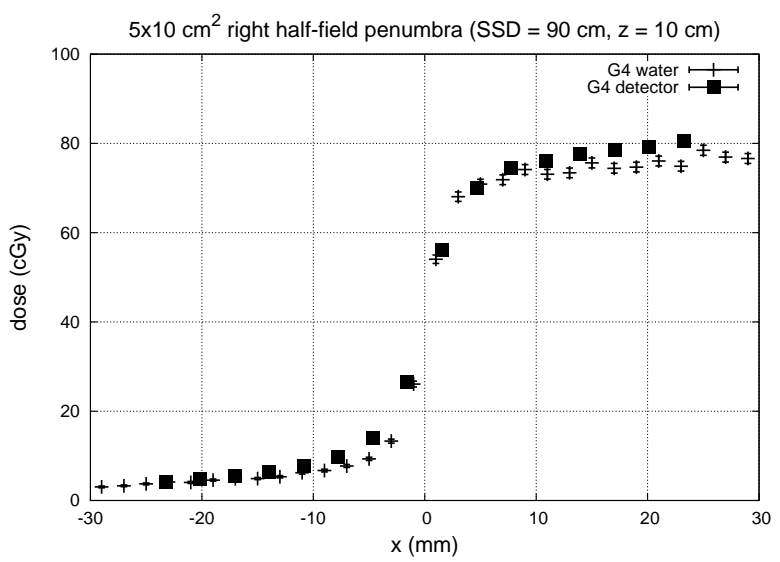

Fig. 6 Geant4 calculations of the penumbra of the halfirradiation field obtained in the water tank (crosses) and in the silicon detector placed in the slab phantom (squares). Error bars represent the statistical uncertainty of the Monte Carlo calculations at $1 \sigma$ level. Symbol size is larger than the error bars for the silicon detector simulation.

irradiation field $5 \times 10 \mathrm{~cm}^{2}$ has been simulated in order to place one of the penumbras of the radiation field at the centre of the crossplane coordinate. In this case, conditions were $S S D=90 \mathrm{~cm}$ and $z=10 \mathrm{~cm}$. Figure 6 represents the penumbra simulated with Geant 4 for both water tank and the silicon detector placed in the water-equivalent slab phantom. The calculations for the silicon detector were renormalized to water-equivalent dose using the result obtained previously. We can see that the penumbra is blurred in the detector simulation with respect to the calculations in water. This must be an effect of the spatial resolution of the detector, given by the width of the strips $(3.1 \mathrm{~mm})$. The statistical uncertainties are similar to those in the previous cases.

Figures 7-9 are devoted to the comparison of the Monte Carlo simulations of the more realistic cylindrical phantom, described in a previous section, with the calculations given by TPS. The treatment head angle (gantry angle) was set to 0 degrees (vertical irradiation) for all the simulations, whereas the cylindrical phantom was rotated to different angles $\left(0^{\circ}\right.$, $45^{\circ}$ and $90^{\circ}$ ) in order to give rise to different orientations of the detector strips with respect to the beam axis. In order to achieve an uniform dose in all the strips, we considered a $10 \times 10 \mathrm{~cm}^{2}$ radiation field, with the geometric centre of the phantom placed at the isocentre. In these cases, we assumed that the material of the entire phantom (including cables and detector) was water, since TPS calculates dose-to-water and Geant4 simulations calculate dose-to-material.

In Fig. 7, differences of approximately $4 \mathrm{cGy}$ between the values calculated by TPS and Geant 4 can be observed. These differences are larger than the uncertainties estimated for TPS $( \pm 0.1$ cGy) and Geant 4 calculations $(0.5 \%, 1 \sigma)$. However, these discrepancies are smaller ( $2 \mathrm{cGy}$ ) when the rotation of the phantom is 45 degrees (shown in Fig. 8), whereas for a rotation of 90 degrees (Fig. 9), the discrepancies are roughly $1 \mathrm{cGy}$. These differences appear because of a misplacement of the sensitive area of the silicon detector. The TPS calculations were made based on a transversal CT image of the phantom, 


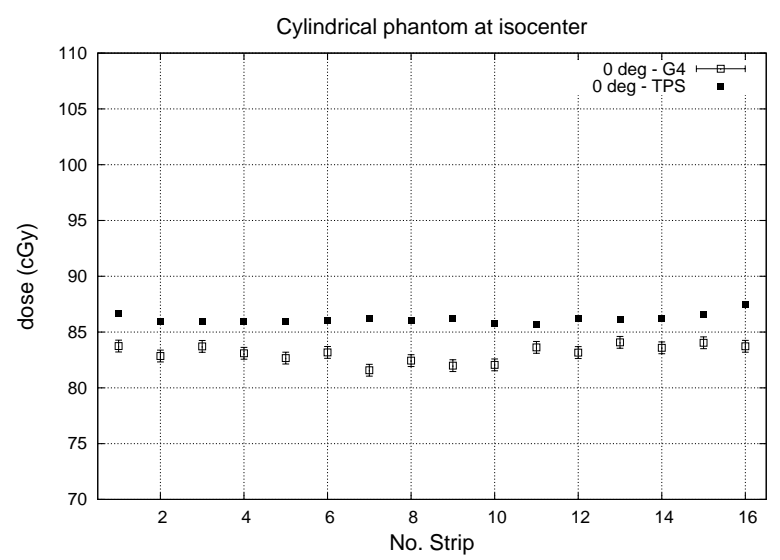

Fig. 7 TPS-calculated (filled squares) and Geant4-simulated (empty squares) dose in water for each strip with phantom rotated 0 degrees. Error bars correspond to the statistical uncertainties of the Monte Carlo results ( $1 \sigma$ level). The uncertainty estimated for the TPS calculations (not represented) was $0.1 \mathrm{cGy}$.

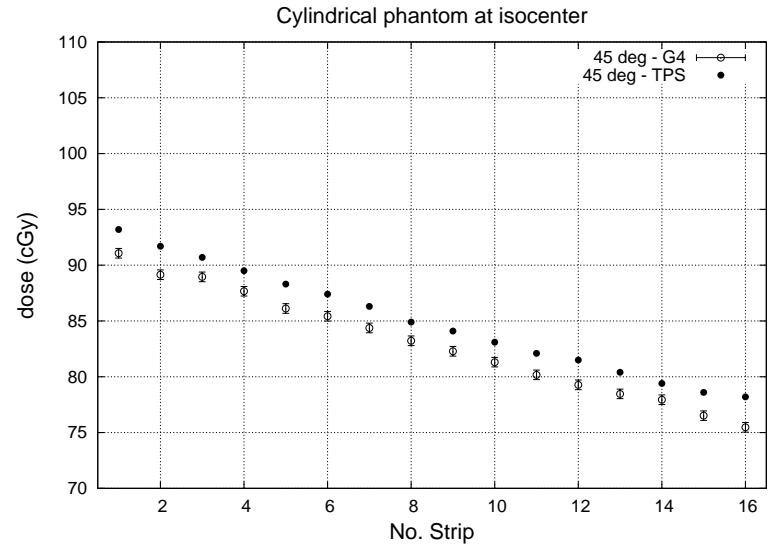

Fig. 8 Same plot as Fig. 7 with the phantom rotated 45 degrees.

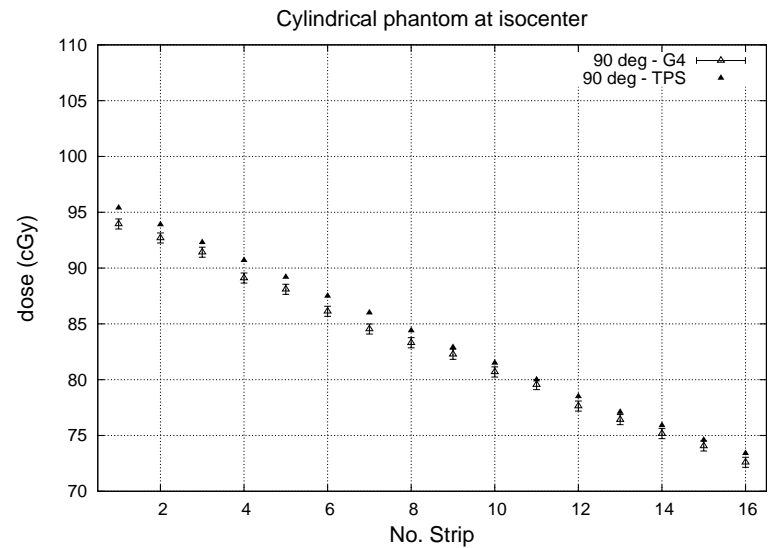

Fig. 9 Same as Fig. 7 with the phantom rotated 90 degrees.

in which it was found that the centre of the detector sensitive area was $3 \mathrm{~mm}$ far from the rotation axis of the cylindrical phantom. This information led us to redesign the phantom in order to correct this effect for future works.

\section{Conclusions}

We have performed Geant4 Monte Carlo simulations of a novel setup for IMRT treatment verification system based on silicon strip detector technology. Monte Carlo simulations have been a very powerful tool for the development of such verification system. We have used Geant 4 simulations as a virtual laboratory which has been useful for us to continue the development of this work and check the suitability of a certain experimental setup. With Monte Carlo simulations we are able to find sources of errors arised in TPS calculations, due to a misplacement of the Si detector sensitive area.

However, despite of some discrepancies, it has been proven the feasibility of this setup. The experimental measurements with the suitable electronics, still in progress, will give us the data needed to study the viability of this prototype.

\section{Acknowledgment}

This work was supported by the Spanish Ministerio de Ciencia e Innovación Contracts FPA2008-04972-C03, FIS2008-04189 and by Instalaciones Inabensa S. A. under the contract 68/83 0214/0129.

\section{References}

1) J. Van Dyk, R. B. Barnett, J. E. Cygler, P. C. Shragge, "Commissioning and quality assurance of treatment planning computers," Int. J. Radiat. Oncol. Biol. Phys., 26, 261-273 (1993).

2) P. Cadman, R. Bassalow, N. P. S. Sidhu, G. Ibbott, A. Nelson, "Dosimetric considerations for validation of a sequential IMRT process with a commercial treatment planning system," Phys. Med. Biol., 47, 3001-3010 (2002).

3) O. A. Zeidan, S. A. L. Stephenson, S. L. Meeks, T. H. Wagner, T. R. Willoughby, P. A. Kupelian, K. M. Langen, "Characterization and use of EBT radiochromic film for IMRT dose verification," Med. Phys., 33, 4064-4072 (2006).

4) A. Niroomand-Rad, C. Blackwel, B. Coursey, K. Gall, J. Galvin, W. McLaughlin, A. Meigooni, R. Nath, J. Rodgers, C. Soares, "Radiochromic film dosimetry: recommendation of AAPM radiation therapy task group 55," Med. Phys., 25[11], 2093-2115 (1998).

5) R. Arráns, H. Miras, M. Ortiz-Seidel, J. A. Terrón, J. Macías, A. Ortiz-Lora, "Radiochromic Film Dosimetry," Rev. Fis. Med., 10[2], 83-104 (2009).

6) I. Redondo-Fernández, C. Buttar, S. Walsh, S. Manolopoulos, J. M. Homer, S. Young, J. Conway, "Performance of the first $\Delta$ OSI microstrip dosimeter prototype in the characterization of a clinical accelerator," Nucl. Instr. Meth. Phys. Res., A573, 141144 (2007).

7) P. S. Nizin, "Electronic equilibrium and primary dose in collimated photon beams," Med. Phys., 20[6], 1721-1729 (1993).

8) S. Agostinelli et al., "GEANT4 - A simulation toolkit," Nucl. Instr. Meth. Phys. Res., A506, 250-303 (2003).

9) J. Allison et al., "Geant4 developments and applications," IEEE Trans. Nucl. Sci., 53[1], 270-278 (2006).

10) P. Rodrigues, A. Trindade, L. Peralta, C. Alves, A. Chaves, M. C. Lopes, "Application of GEANT4 radiation transport toolkit to dose calculations in anthropomorfic phantoms," Appl. Radiat. Isot., 61, 1451-1461 (2004).

11) E. Poon, J. Seuntjens, F. Verhaegen, "Consistency test of the electron transport algorithm in the GEANT4 Monte Carlo code," Phys. Med. Biol., 50, 681-694 (2005). 
12) J. Tinslay, B. Faddegon, J. Perl, M. Asai, "Verification of Bremsstrahlung Splitting in Geant 4 for Radiotherapy Quality Beams," Med. Phys., 34, 2504 (2007).

13) B. A. Faddegon, M. Asai, J. Perl, C. Ross, J. Sempau,J. Tinslay, F. Salvat, "Benchmarking of Monte Carlo simulation of bremsstrahlung from thick targets at radiotherapy energies," Med. Phys., 35, 4308-4317 (2008).

14) S. Larsson, R. Svensson, I. Gudowska, V. Ivanchenko, A. Brahme, "Radiation transport calculations for $50 \mathrm{MV}$ photon therapy beam using the Monte Carlo code GEANT4," Radiat. Prot. Dosim., 115[1-4], 503-507 (2005).

15) H. Paganetti, H. Jiang, S. Y. Lee, H. M. Kooy, "Accurate Monte Carlo simulations for nozzle design, commissioning and quality assurance for a proton radiation therapy facility," Med. Phys., 31, 2107-2118 (2004).

16) H. Paganetti, H. Jiang, K. Parodi, R. Slopsema, M. Engelsman, "Clinical implementation of full Monte Carlo dose calculation in proton beam therapy," Phys. Med. Biol., 53, 4825-4853 (2008).

17) http://geant4.web.cern.ch/geant4/UserDocumentation/

18) S. Chauvie, S. Guatelli, V. Ivanchenko, F. Longo, F. Mantero, B. Mascialino, P. Nieminen, L. Pandola, S. Parlati, L. Peralta, M. G. Pia, M. Piergentili, P. Rodrigues, S. Saliceti, A. Trindale, "GEANT4 low energy electromagnetic physics," Proceedings of the International Conference on Computing in High Energy and Nuclear physics, CHEP2001, Beijing, China, September 3-7, 2001.

19) S. Chauvie, S. Guatelli, V. Ivanchenko, F. Longo, F. Mantero, B. Mascialino, P. Nieminen, L. Pandola, S. Parlati, L. Peralta, M. G. Pia, M. Piergentili, P. Rodrigues, S. Saliceti, A. Trindale,
"Geant4 Low Energy Electromagnetic Physics," IEEE Nuclear Science Symposium 2004 Conference Record, Vol. 3, 18811885, 2004.

20) D. E. Cullen, J. H. Hubbell, L. Kissel, EPDL97: the Evaluated Photon Data Library, '97 version, UCRL-50400, vol. 6, rev. 5, Lawrence Livermore National Laboratory (LLNL) (1991).

21) S. T. Perkins, D. E. Cullen, S. M. Seltzer, Tables and Graphs of Electron-Interaction Cross-Sections from $10 \mathrm{eV}$ to $100 \mathrm{GeV}$ Derived from the LLNL Evaluated Electron Data Library (EEDL), $Z=1-100$, UCRL-50400, vol. 31, Lawrence Livermore National Laboratory (LLNL) (1991).

22) S. T. Perkins, D. E. Cullen, M. H. Chen, J. H. Hubbell, J. Rathkopf, J. Scofield, Tables and Graphs of Atomic Subshell and Relaxation Data Derived from the LLNL Evaluated Atomic Data Library (EADL), Z=1-100, UCRL-50400, vol. 30, Lawrence Livermore National Laboratory (LLNL) (1991).

23) H. Burkhardt, V. M. Grichine, V. N. Ivanchenko, P. Gumplinger, R. P. Kokoulin, M. Maire, L. Urban, "Geant4 'standard' electromagnetic physics package," Proceedings MC2005, Chattanooga, Tennessee, April 17-21, 2005, on CD-ROM, American Nuclear Society, LaGrange Park, II (2005).

24) M. A. Cortés-Giraldo, J. M. Quesada, M. I. Gallardo, "Geant4 Application for the Simulation of the Head of a Siemens Primus Linac," AIP Conf. Proc., 1231 209-210 (2009).

25) http://www-nds.iaea.org/phsp

26) B. R. B. Walters, I. Kawrakow, D. W. O. Rogers, "History by history statistical estimators in the BEAM code system," Med. Phys., 29, 2745-2752 (2002). 